\title{
Are CVD Patients Under Oxidative Stress?
}

\author{
Yedidya Dotan, Dov Lichtenberg and Ilya Pinchuk \\ Dept. of Physiology and Pharmacology, Sackler Medical School, Tel Aviv University \\ Israel
}

\section{Introduction}

Oxidative stress has long been associated with cardiovascular disease (CVD) (Abuja \& Albertini, 2001; Halliwell \& Gutteridge, 1990; Parthasarathy et al., 2001; Steinbrecher et al., 1984). It was even assumed that the prevalence of CVD alone indicates the prevalence of oxidative stress (Witztum, 1994). Moreover, cross-sectional studies indicated that supplementation of low molecular weight antioxidants is associated with a relatively low incidence of CVD (Jha et al., 1995). By contrast, in most of the interventional studies the antioxidant supplementation did not prevent the progression of CVD nor did it improve any of the many clinical endpoints (Shekelle et al., 2004; Vivekananthan et al., 2003; Miller 2005; Bjelakovic, 2007; Dotan, 2009a; Dotan, 2009b). Based on these findings, Witztum (Witztum, 1998) and Morrow (Morrow, 2003) hypothesized that only individuals under oxidative stress may benefit from antioxidant supplementation. This, of course, implies that only people under oxidative stress should be treated with antioxidants. This, in turn, means that a criterion must be established for the ill-defined, intuitively understood term "oxidative stress".

This issue is of special importance in light of our previous study that demonstrated that no single index can be used as a universal criterion, indicating that there are several types of oxidative stress (Dotan et al., 2004). Hence, the question remains which criterion (or criteria) can be used to identify who is likely to benefit from antioxidant supplementation. The answer to this question can, theoretically, be based either on a criterion for the relevant type of oxidative stress (e.g. lipid peroxidation, as assessed by the concentrations of MDA or isoprostanes) or/and on diagnosis of specific diseases for which there is sufficient evidence for benefit of antioxidant supplementation.

In the current study, we present the results of a meta-analysis of case-control studies used to assess the association between CVD and oxidative stress, as evaluated on the basis of different criteria. Unlike previous meta-analyses, we analyzed the association between CVD and criteria for each of the types of OS. We hope that eventually this analysis will enable us to define threshold values of relevant indices of the relevant type of oxidative stress for treatment with antioxidants (Deeks, 2001; Deeks \& Altman, 2004).

\section{Methods}

This work was performed according to the guidelines outlined by the Meta-analysis Of Observational Studies in Epidemiology (MOOSE) group (Stroup et al., 2000). A detailed 
description of the data retrieval process, the selection criteria and the analyses of the data is given in the following subsections.

\subsection{Retrieval and selection of studies to be included in the analysis}

First, we had to define criteria to be used in our search for clinical trials to be included in our meta-analysis (Fig. 1). The following criteria were defined for our search:

i. Design: only case-control or nested case control studies were included.

ii. Methods: for a clinical study to be included in our meta-analysis it had to use only generally accepted methods for the evaluation of oxidative stress (Dotan et al., 2004).

iii. Size: to be included in our meta-analysis, the study had to involve at least $20 \mathrm{CVD}$ patients and at least 20 "CVD-free" individuals, serving as a control group.

The latter demand requires further definition of the two populations that can be regarded as being "CVD patients" and their respective controls. We included in the control group only "pathology-free" individuals. To be included in the group of "CVD patients", a person had to be diagnosed with one of the following conditions: (i) either stable angina pectoris (STP) or unstable angina pectoris (UTP), diagnosed either by angiogram or by physical examination, (ii) patients suffering from any of the stages of coronary stenosis and (iii) a recorded history of CVD (e.g. myocardial infraction).

With these criteria in mind, we searched through two major databases, namely, the Medline database (http://www.ncbi.nlm.nih.gov/entrez/query.fcgi?CMD=search\&DB=pubmed) and the Thomson Reuters ISI Web of Science. Reference mining was conducted to find an arbitrarily predefined number of studies (20) that fit all the above selection criteria. We defined a list of keywords to search and conducted a simple keyword search, similar to that described by Shekelle et al. (Shekelle et al., 2004).

Data of case-control studies may be biased towards the prevailing paradigm and the findings of other recent trials (French et al., 2005; Moher \& Tsertsvadze, 2006). Such bias must be considered when conducting a search for studies to be included in meta-analyses because it may cause a change in the significance of the pooled variables (French et al., 2005; Moher \& Tsertsvadze, 2006). This is of special importance in the present meta-analysis because the prevailing paradigm changed from viewing "oxidative stress" as a cause of atherosclerosis (Witztum, 1994) to viewing it as being merely a result of questionable significance (Witztum \& Steinberg, 2001). In addition, we had to assess the completeness of the retrieved data and its validity with respect to the complete body of published data (Bennett et al., 2004). Towards this end, we have devised an algorithm similar to the "capture-recapture technique" proposed by Spoor et al. (Spoor et al., 1996). Spoor et al. used different methods of data retrieval to assess their results. We used, for the same purpose, different time frames. Briefly, to achieve as random as possible subset of studies (Furukawa et al., 2002), we searched the Medline database to identify studies published between 1.1.1990 and 1.8.2003 and used studies dating from 1.8.2003 until 1.2.2006 to reassert our findings, as proposed by Moher \& Tsertsvadze (Moher \& Tsertsvadze, 2006). By that, we have minimized the bias towards recent publications.

\subsection{Data analysis}

The difference between individuals with prevalent CVD and their controls, as observed in each of the selected studies, was expressed in terms of two factors: (i) the standard mean difference (SMD), which is a composite index, and (ii) the $95 \%$ confidence interval of the 
SMD, which reflects both the mean difference and the standard deviation. Our study required the use of SMD for two reasons: (i) it allows pooling of results obtained from different studies and (ii) its use compensates, at least partially, for the use of different methods, different units of measurement and inter-lab differences (Deeks et al., 2001). We implemented SMD by using "Hedges' adjusted g" because it contains a correction for small sample bias (Bennett et al., 2004).

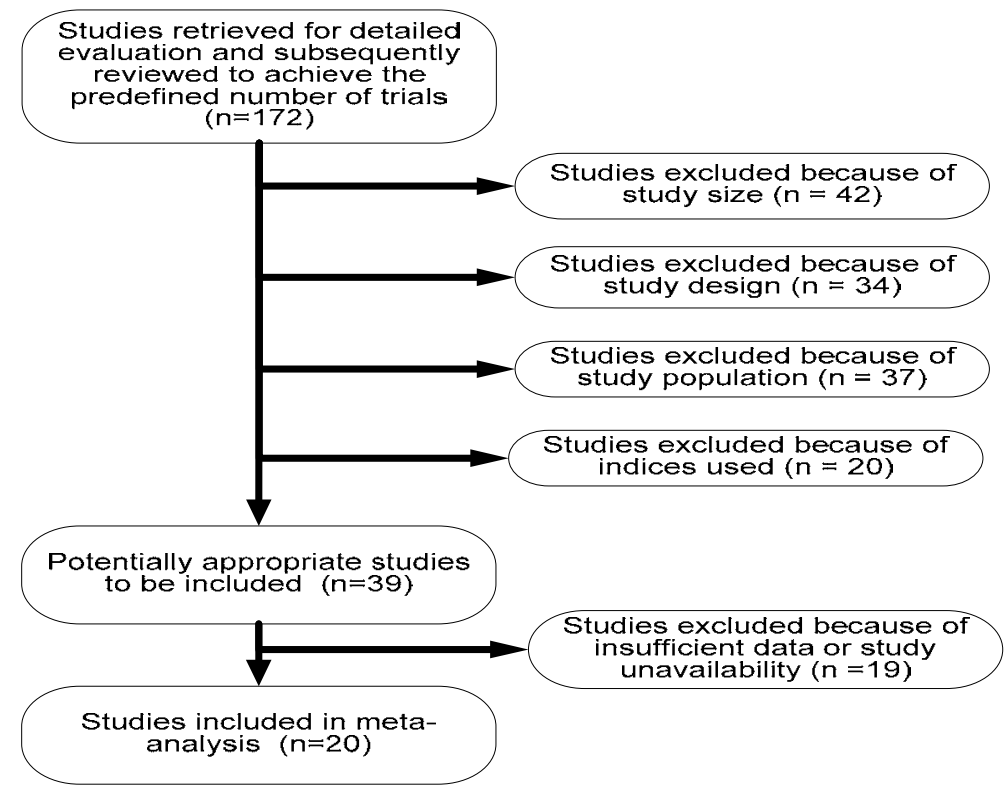

Fig. 1. An outline of the flow of studies in the meta-analyses. We identified many publications that contained the required sets of keywords (e.g. [CVD or CHD] and oxidative stress).

To pool our results, we have considered two possible models, namely the "fixed effect model", which assumes that all the studies and trials are the repeating of the same experiment (Lau et al., 1998) or the "random effects model" of DerSimonian and Laird, which assumes that the clinical trials estimate a different, yet related, variables with a common distribution (Higgins et al., 2002; Lau et al., 1998). From these two models, we chose to use the random effects model, because we expected heterogeneous results from observational studies (Egger et al., 1998). In our view, the "fixed effect model" oversimplifies the problem and its accuracy has been questioned by others in the same context (Lau et al., 1998), particularly when there was a need to pool results of heterogeneous populations (Lau et al., 1998).

In our analyses of the data, we relate not only to the mean value of the pooled variables. We also used the collected data to assess the credibility of the results as previously proposed by Smith et al. (Smith et al., 1997). Assessment of heterogeneity in a meta-analysis of observational studies is as important as the mean results (Egger et al., 1998; Egger et al., 1997a). We assessed the heterogeneity in terms of the following indices as proposed by Song 
(Song et al., 2001): (i) $\chi^{2}$ (Deeks et al., 2001), (ii) $i^{2}$ (used by the RevMan 4.2.8 software) and (iii) $\log \left(\mathrm{X}^{2} / \mathrm{DF}+1\right)$, where DF is the number of degrees of freedom (Deeks et al., 2001), for comparing the degree of heterogeneity between heterogeneous results.

To detect variables that affect our models, we used stratification, clustering of associated variables and meta-regression models (Smith et al., 1997; Song et al., 2001). Yet, unlike in the case of clinical trials, we analyzed either the complete trial or a specific subgroup within the trial (Lau et al., 1998). We also analyzed the correlations between indices of oxidative stress and various stages of CVD, to determine causality (Egger et al., 1998; Mulrow, 1994; Smith et al., 1997). To assess the effects of exposure variables on the outcome, we used stratification for common risk-related variables such as age (in subgroups), gender and history of smoking (Smith et al., 1997; Sterne, et al., 2001). We also used stratification for variables specific to the pathology (e.g. stable and unstable angina pectoris in CVD), as proposed by Smith et al (Smith et al., 1997). We used meta-regression (logistic and multiple linear) to model the increase in continuous and ordinal SMD, as well as the annual event rates (Lau et al., 1998). We used the observed SMD as our dependent variable and the covariates of interest (independent variables) to assess the sources of heterogeneity (Lau et al., 1998). A mixed stepwise regression was used to detect trends and factors affecting SMD of MDA concentration in CVD (Lau et al., 1998).

To detect (and assess) the degree of bias in our meta-analyses, we have used both the "Trim and Fill method" (Duval \& Tweedie , 2000) and a simple funnel plots (Sutton et al., 2000). A funnel plot is a regression of each trial's effect size against a measure of its size (e.g. 1/standard error) (Sutton et al., 2000). An asymmetry in a forest plot is attributed to the high probability that smaller studies with less statistical power are not published. Asymmetric publications were "trimmed" and then the number of studies missing was calculated. We used a simulation to fill the missing studies as described by Duval \& Tweedie (Duval \& Tweedie, 2000), thus verifying our initial assumption. We used additional methods to assess the relations between study size and results, mainly because publication-bias is not the only reason for asymmetry in funnel plots, and because this method may be subjective and have a relatively high false-positive rate of detecting bias (Sterne et al., 2001). We compensated for the subjective nature of graphical assessment by using the rank correlation method (Thornton and Lee, 2000) and an adjustment of the "Egger's method" (Egger et al., 1997b). Rank correlation is the statistical analogue of the funnel plot, namely a regression of the effect size (SMD) against both sizes of study and 1/SE (Thornton and Lee, 2000). We used an adjustment of the "Egger's method" to detect publication bias and its direction. We defined the standard normal deviate (SND) as the mean difference, divided by its standard error regressed against the estimate's precision (Bennett et al., 2004). We defined the threshold pvalue for the intercept at a value of 0.1 to detect possible bias (Bennett et al., 2004).

\section{Results}

As depicted in Fig. 1, we reviewed 172 studies, of which 20 were selected for analysis. These chosen 20 studies compared 1068 CVD patients to 2128 matched controls, using 15 common indices to assess oxidative stress. A summary of the results is given in Fig. 2 (Akkus et al., 1996; Cavalca et al., 2001; Chiu et al., 1994; Cipollone et al., 2000; Clejan et al., 2002; Delanty et al., 1997; Durak et al., 2001; Ferns et al., 2000; Gackowski et al., 2001; Haidari et al., 2001; Halevy et al., 1997; Karmansky et al., 1996; Kesavulu et al., 2001; Kostner et al., 1997; 
McMurray et al., 1992; Schisterman et al., 2002; Singh et al., 1995; Tamer et al., 2002; Turgan et al., 1999; Weinbrenner et al., 2003).

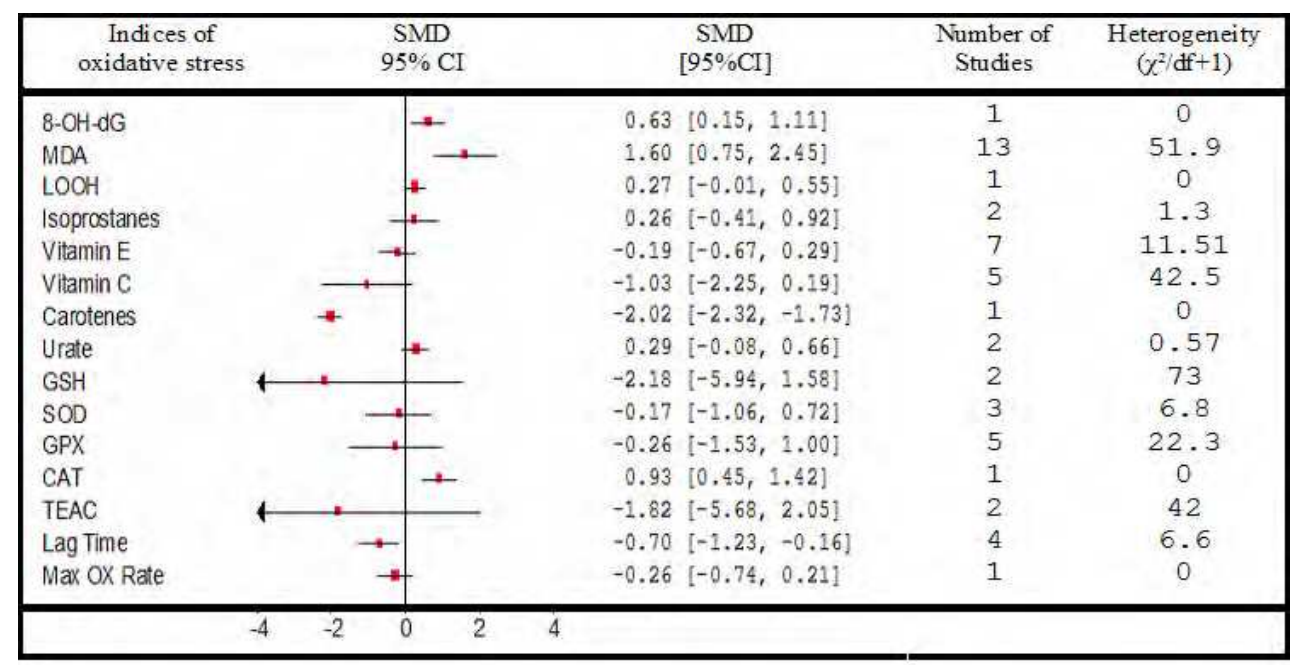

Fig. 2. Indices of oxidative stress in cardiovascular disease. We present both graphically and numerically the pooled standardized mean difference (SMD) and the $95 \%$ confidence interval. Also given is the heterogeneity, as defined by $x^{2} / \mathrm{df}+1$, of the studies regarding each index. Note that MDA is the only accepted index of oxidative stress that shows a mean difference greater the 1SMD.

Given the prevailing paradigm regarding the association between atherosclerosis and lipid peroxidation (Witztum \& Steinberg, 2001), it is not surprising that MDA was the most frequently used index utilized to assess oxidative stress (13/20 studies). Furthermore, only MDA exhibited a strong effect size, given by a SMD of 1.60 (0.75 to 2.45). Using the "Trim and Fill" method (Duval \& Tweedie, 2000) revealed that at least three studies were missing from the funnel plot. Adjusting for the latter finding resulted in a much lower SMD of 0.71 (0.37 to 1.05). The "Egger method" (Egger et al., 1997b) identified an intercept that was significant and positive, supporting the conclusion that the previously observed differences were overestimated.

We performed a mixed stepwise regression for MDA concentrations, factoring in age, gender, smoking habits, severity of CVD, prevalence of hypertension and diabetes mellitus as well as the use of NSAIDs. This analysis indicated that the inclusion of smokers in both CVD patients and control groups (estimate ${ }^{1}=-1.3, p=0.04$ ), results in an underestimation of the association between CVD and oxidative stress. By contrast, the inclusion of patients with both acute (severe CVD) and chronic (mild CVD) coronary syndromes (estimate =3.1, $\mathrm{p}=0.01$ ), results in an overestimation of the latter associations.

We stratified the results from those studies that assessed MDA concentrations into three groups: (i) patients with unstable angina pectoris (UAP), (ii) patients with stable angina

${ }^{1}$ Estimate: A numerical value obtained from a statistical sample and assigned to a population parameter. 
pectoris (SAP) and (iii) healthy controls. As seen in Fig. 3, the MDA concentrations of patients with SAP are not different from matched controls, whereas UAP patients have significantly higher MDA concentrations than both healthy controls and patients with SAP. We also stratified the results from two types of studies, those that had acute coronary conditions (MI, UAP) and those CVD patients who had chronic coronary conditions (SAP and occlusions). As seen in Fig. 4, patients with acute coronary conditions had marked and significantly higher MDA concentrations $(S M D=2.30,0.93$ to 3.67) than matched controls. By contrast, patients with chronic coronary conditions had only slightly higher MDA concentrations (SMD $=0.60,0.18$ to 1.01 ) as compared with matched controls.

The concentrations of beta-carotene, as evaluated by Singh et al. (Singh et al., 1995), were significantly lower in CVD patients than in matched controls. The difference between the concentrations all other low molecular weight antioxidants (LMWA) in patients with CVD and healthy controls were not statistically significant (Fig. 2). In six of the seven studies vitamin E concentration in CVD patients and controls was similar (SMD $=0.02,-0.17$ to 0.21 ).

Significantly lower concentrations were observed only by Singh et al. Furthermore, pooling the results from all the seven studies failed to achieve statistically significant differences (SMD $=-0.19,-0.67$ to 0.29$)$, although the heterogeneity index substantially increased from 1.35 to 11.51. The differences between the results of Singh et al. and all other studies cannot be attributed either to publication bias or to small-sample bias. A viable possibility is that the difference is due to selection bias, particularly by a biased selection of controls. Indeed, the major difference between the results obtained by Singh et al. and the results observed in all the other studies assessing LMWA was that Singh et al. selected their control group to exclude most, if not all of the CVD-related risk factors (smoking, DM and glucose intolerance), whereas the CVD patients were not devoid of these risk factors.

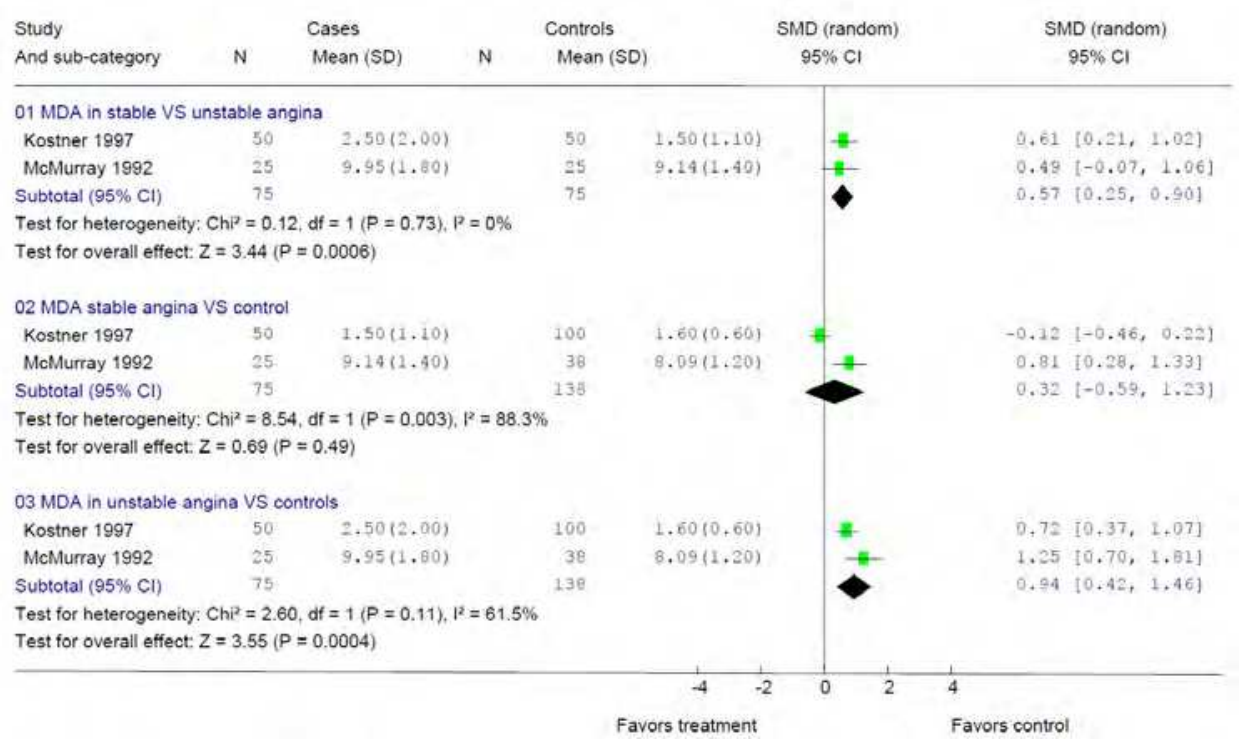

Fig. 3. A forest plot of studies assessing the differences between patients with stable angina pectoris and patients with unstable angina pectoris (upper panel) and comparison of each of these groups with controls (two lower panels). 
Unfortunately, those studies that used either DNA damage or total antioxidant capacity (TEAC) to assess oxidative stress in patients with CVD in comparison to healthy controls (Fig. 2) were too few and too small to enable any conclusions.

\section{Discussion}

Based on many lines of indirect evidence, oxidative stress has long been associated with CVD. The following findings have been considered to lend support to the oxidative theory of CVD: (i) oxidized LDL may cause formation of foam cell in-vitro (Chisolm \& Steinberg, 2000), (ii) the development of atherosclerosis is preceded by an increase of the levels of many indicators of oxidative stress in lab animals (Chisolm \& Steinberg, 2000) and (iii) the incidence of CVD in individuals with low concentrations of antioxidants is relatively high (Chisolm and Steinberg, 2000). These (and other) findings and clinical trials led researchers to two assumptions: (i) oxidative stress plays a pivotal role in the formation of atherogenic plaque and (ii) individuals with prevalent CVD are likely to be under oxidative stress and therefore have high plasma concentrations of lipid peroxidation products and loss of LMWA.

The first assumption has long been disputed and is currently under scrutiny (Williams \& Fisher, 2005). The second assumption is weakened by the results of our current metaanalysis, which shows that the prevalence of CVD is only slightly associated with OS, as defined on the basis MDA concentration. By contrast, no evidence is available for association between CVD and OS, as determined on the basis of all other indices of oxidative stress. Specifically, the concentrations of almost all the micronutrients in the plasma of CVD patients are within normal ranges (Fig. 2), the activities of relevant enzymes did not differ from those observed in matched controls (Fig. 2). The same results were observed for most of the indices of lipid peroxidation. The only index that is significantly different with the prevalence of CVD is the plasma concentration of MDA. Even if we choose to ignore the reservations regarding the use of MDA as an index of lipid peroxidation (Draper et al., 1993), the possibility of publication bias should not be ignored. Hence, we think that the existing evidence for association between oxidative stress and the prevalence of CVD is quite weak. In other words, it appears that the role of oxidative stress in atherogenesis has been overestimated. In accordance with this conclusion, is the viewpoint of the recent review, regarding association of several more OS indices (circulation levels of oxidized LDL and myeloperoxidase) with CVD (Strobel et al., 2011). The authors conclude that "results of studies using Ox-LDL have been equivocal" and that "the ability of oxidative stress biomarkers to predict CVD has yet to be established".

The latter considerations accord with the current trend in cardiovascular disease research, which views atherosclerosis as mostly an inflammatory disease (Ross, 1999) and implies that oxidative stress is a result and not the cause for atherosclerosis. This trend also accords with and therefore is strengthened by two of our findings: (i) we observed that patients with UAP, a condition commonly associated with acute inflammation, are under higher "oxidative stress", as assessed by the serum concentration of MDA, than both patients with SAP and matched controls (Fig. 3) and (ii) we observed that studies including more acute conditions are under higher "oxidative stress" than matched controls (Fig. 4).

In our opinion, these findings are of little relevance to the possibility that many individuals may benefit from antioxidant supplementation. 
Our ongoing research is aimed at developing such an assay (or assays) that can serve as a basis for selective antioxidant supplementation. Our working hypothesis is a two step approach of identifying those individuals that may benefit from antioxidant supplementation. The first step is an initial, short-term treatment with vitamin E. The second step should be to assess plasma concentrations of lipid peroxidation products and to continue treatment with vitamin E only to individuals, responding to treatment by reducing significantly plasma concentration of lipid peroxidation products. In conclusion, we have no evidence that justify indiscriminate supplementation of vitamin E, nor do we have sufficient evidence to ban it, as recommended by the authors of the Cache County Study (Hayden et al., 2007). At present we have no assay that can be used to identify patients that are likely to benefit from Vitamin E supplementation.

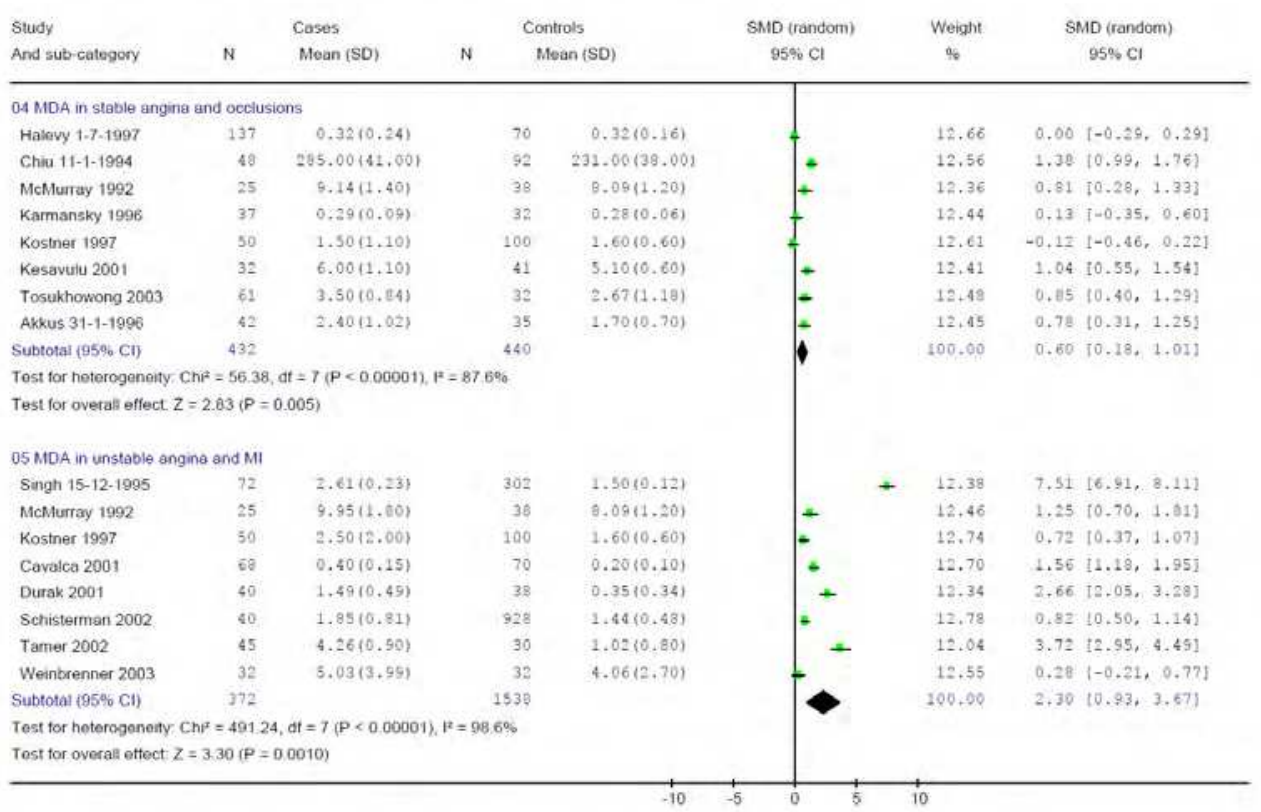

Fig. 4. A forest plot comparing two strata of studies. The first stratum compares the differences between patients with either stable angina pectoris or mild occlusions of the coronary arteries and healthy controls. The second stratum compares the differences between patients with either unstable angina pectoris or MI and healthy controls. Notably, while the first stratum of patients with SAP varies slightly from healthy controls, the second stratum of patients with UAP shows a significant difference. The two strata differ significantly $(\mathrm{p}=0.02)$.

\section{Conclusion}

Our meta-analysis shows that the commonly accepted paradigm regarding the role of OS in the pathogenesis of CVD appears to be overestimated. CVD is associated with OS only when the evaluation of OS is based on plasma concentrations of MDA. Notably, even this 
association is questionable due to (i) poor reliability of the laboratory assay of MDA and (ii) publication bias.

Most of the clinical trials that were designed to reduce OS by means of antioxidant supplementation yielded disappointing results. The latter results are consistent with the results of our meta-analysis. Hence, oxidative stress is only weakly associated with the prevalence of cardiovascular disease.

\section{Acknowledgments}

We thank the Lady Davis foundation and Israel Science Foundation (research grant 362/0218) for financial support. We would also like to thank Dr. Yariv Gerber and Prof. Uri Goldburt for their sound guidance and Mrs. Ariela Bor for her skillful technical assistance.

\section{References}

Abuja, P.M. and Albertini, R., 2001. Methods for monitoring oxidative stress, lipid peroxidation and oxidation resistance of lipoproteins. Clin.Chim.Acta 306, 1-17.

Akkus, I., Saglam, N.I., Caglayan, O., Vural, H., Kalak, S., and Saglam, M., 1996. Investigation of erythrocyte membrane lipid peroxidation and antioxidant defense systems of patients with coronary artery disease (CAD) documented by angiography. Clin.Chim.Acta 244, 173-180.

Bennett, D.A., Latham, N.K., Stretton, C., and Anderson, C.S., 2004. Capture-recapture is a potentially useful method for assessing publication bias. Journal of Clinical Epidemiology 57, 349-357.

Bjelakovic, G., Nikolova D., Gluud L.L. et al, 2007. Mortality in randomized trials of antioxidant supplements for primary and secondary prevention: systematic review and meta-analysis. JAMA 297, 842- 857.

Cavalca, V., Cighetti, G., Bamonti, F., Loaldi, A., Bortone, L., Novembrino, C., De Franceschi, M., Belardinelli, R., and Guazzi, M.D., 2001. Oxidative stress and homocysteine in coronary artery disease. Clin.Chem. 47, 887-892.

Chisolm, G.M. and Steinberg, D., 2000. The oxidative modification hypothesis of atherogenesis: an overview. Free Radical Biology and Medicine 28, 1815-1826.

Chiu, H.C., Jeng, J.R., and Shieh, S.M., 1994. Increased oxidizability of plasma low density lipoprotein from patients with coronary artery disease. Biochim.Biophys.Acta 1225, 200-208.

Cipollone, F., Ciabattoni, G., Patrignani, P., Pasquale, M., Di Gregorio, D., Bucciarelli, T., Davi, G., Cuccurullo, F., and Patrono, C., 2000. Oxidant Stress and AspirinInsensitive Thromboxane Biosynthesis in Severe Unstable Angina. Circulation 102, 1007.

Clejan, S., Japa, S., Clemetson, C., Hasabnis, S.S., David, O., and Talano, J.V., 2002. Blood histamine is associated with coronary artery disease, cardiac events and severity of inflammation and atherosclerosis. J Cell Mol.Med 6, 583-592.

Deeks, J.J., 2001. Systematic reviews in health care: Systematic reviews of evaluations of diagnostic and screening tests. BMJ 323, 157-162.

Deeks, J.J. and Altman, D.G., 2004. Diagnostic tests 4: likelihood ratios. BMJ 329, 168-169.

Deeks, J.J., Altman, D.G., and Bradburn, M.J., 2001. Statistical methods for examining heterogeneity and combing results from several studies in meta-analysis. 
Systematic reviews in health care Meta-analysis in context, Second ed. BMJ Publishing Group , pp. 285-312.

Delanty, N., Reilly, M.P., Pratico, D., Lawson, J.A., McCarthy, J.F., Wood, A.E., Ohnishi, S.T., Fitzgerald, D.J., and FitzGerald, G.A., 1997. 8-Epi PGF2\{alpha\} Generation During Coronary Reperfusion: A Potential Quantitative Marker of Oxidant Stress In Vivo. Circulation 95, 2492.

Dotan, Y., Lichtenberg, D., and Pinchuk, I., 2004. Lipid peroxidation cannot be used as a universal criterion of oxidative stress. Progress in Lipid Research 43, 200-227.

Dotan, Y., Lichtenberg, D., Pinchuk, I., 2009a. No evidence supports vitamin E indiscriminate supplementation. BioFactors 35, 469-473.

Dotan, Y., Pinchuk, I., Lichtenberg, D., Leshno, M., 2009b. Decision analysis supports the paradigm that indiscriminate supplementation of vitamin $\mathrm{E}$ does more harm than good. ATVB 29, 1304-1309.

Draper, H.H., Squires, E.J., Mahmoodi, H., Wu, J., Agarwal, S., and Hadley, M., 1993. A comparative evaluation of thiobarbituric acid methods for the determination of malondialdehyde in biological materials. Free Radic.Biol.Med. 15, 353-363.

Durak, I., Kacmaz, M., Cimen, M.Y., Buyukkocak, U., and Ozturk, H.S., 2001. Blood oxidant/antioxidant status of atherosclerotic patients. Int.J.Cardiol. 77, 293-297.

Duval, S. and Tweedie, R., 2000. Trim and Fill: A Simple Funnel-Plot-Based Method of Testing and Adjusting for Publication Bias in Meta-Analysis. Biometrics 56, 455463.

Egger, M., Schneider, M., and Smith, G.D., 1998. Meta-analysis Spurious precision? Metaanalysis of observational studies. BMJ 316, 140-144.

Egger, M., Smith, G.D., and Phillips, A.N., 1997a. Meta-analysis: Principles and procedures. BMJ 315, 1533-1537.

Egger, M., Smith, G.D., Schneider, M., and Minder, C., 1997b. Bias in meta-analysis detected by a simple, graphical test. BMJ 315, 629-634.

Ferns, G., Williams, J., Forster, L., Tull, S., Starkey, B., and Gershlick, A., 2000. Cholesterol standardized plasma vitamin E levels are reduced in patients with severe angina pectoris. Int.J Exp Pathol. 81, 57-62.

French, S., McDonald, S., McKenzie, J., and Green, S., 2005. Investing in updating: how do conclusions change when Cochrane systematic reviews are updated? BMC Medical Research Methodology 5, 33.

Furukawa, T.A., Guyatt, G.H., and Griffith, L.E., 2002. Can we individualize the 'number needed to treat'? An empirical study of summary effect measures in meta-analyses. Int.J.Epidemiol. 31, 72-76.

Gackowski, D., Kruszewski, M., Jawien, A., Ciecierski, M., and Olinski, R., 2001. Further evidence that oxidative stress may be a risk factor responsible for the development of atherosclerosis. Free Radic.Biol.Med. 31, 542-547.

Haidari, M., Javadi, E., Kadkhodaee, M., and Sanati, A., 2001. Enhanced Susceptibility to Oxidation and Diminished Vitamin E Content of LDL from Patients with Stable Coronary Artery Disease. Clin Chem 47, 1234.

Halevy, D., Thiery, J., Nagel, D., Arnold, S., Erdmann, E., Hofling, B., Cremer, P., and Seidel, D., 1997. Increased Oxidation of LDL in Patients With Coronary Artery Disease Is Independent From Dietary Vitamins E and C. Arterioscler Thromb Vasc Biol 17, 1432. 
Halliwell, B. and Gutteridge, J.M., 1990. Role of free radicals and catalytic metal ions in human disease: an overview. Methods Enzymol. 186, 1-85.

Hayden, K.M., Welsh-Bohmer, K.A., Wengreen, H.J., Zandi, P.P., Lyketsos, C.G., and Breitner, J.C.S., 2007. Risk of Mortality with Vitamin E Supplements: The Cache County Study. The American Journal of Medicine 120, 180-184.

Higgins, J., Thompson, S., Deeks, J., and Altman, D., 2002. Statistical heterogeneity in systematic reviews of clinical trials: a critical appraisal of guidelines and practice. J Health Serv.Res Policy 7, 51-61.

Jha, P., Flather, M., Lonn, E., Farkouh, M., and Yusuf, S., 1995. The Antioxidant Vitamins and Cardiovascular Disease: A Critical Review of Epidemiologic and Clinical Trial Data. Ann Intern Med 123, 860-872.

Karmansky, I., Shnaider, H., Palant, A., and Gruener, N., 1996. Plasma Lipid Oxidation and Susceptibility of Low-Density Lipoproteins to Oxidation in Male Patients With Stable Coronary Artery Disease. Clinical Biochemistry 29, 573-579.

Kesavulu, M.M., Rao, B.K., Giri, R., Vijaya, J., Subramanyam, G., and Apparao, C., 2001. Lipid peroxidation and antioxidant enzyme status in Type 2 diabetics with coronary heart disease. Diabetes Res.Clin.Pract. 53, 33-39.

Kostner, K., Hornykewycz, S., Yang, P., Neunteufl, T., Glogar, D., Weidinger, F., Maurer, G., and Huber, K., 1997. Is oxidative stress causally linked to unstable angina pectoris? A study in 100 CAD patients and matched controls. Cardiovasc.Res. 36, 330-336.

Lau, J., Ioannidis, J.P., and Schmid, C.H., 1998. Summing up evidence: one answer is not always enough. The Lancet 351, 123-127.

McMurray, J., Chopra, M., Abdullah, I., Smith, W.E., and Dargie, H.J., 1992. Evidence for oxidative stress in unstable angina. Br.Heart J 68, 454-457.

Miller, E.R. III, Pastor-Barriuso, R., Dalal, D. et al., 2005. Meta-analysis: high-dosage vitamin E supplementation may increase all-cause mortality. Ann Intern Med 142, 37- 46.

Moher, D. and Tsertsvadze, A., 2006. Systematic reviews: when is an update an update? The Lancet 367, 881-883.

Morrow, J.D., 2003. Is Oxidant Stress a Connection Between Obesity and Atherosclerosis? Arterioscler Thromb Vasc Biol 23, 368-370.

Mulrow, C.D., 1994. Systematic Reviews: Rationale for systematic reviews. BMJ 309, 597.

Parthasarathy, S., Khan-Merchant, N., Penumetcha, M., and Santanam, N., 2001. Oxidative stress in cardiovascular disease. J Nucl.Cardiol. 8, 379-389.

Ross, R., 1999. Atherosclerosis -- An Inflammatory Disease. N Engl J Med 340, 115-126.

Schisterman, E.F., Faraggi, D., Browne, R., Freudenheim, J., Dorn, J., Muti, P., Armstrong, D., Reiser, B., and Trevisan, M., 2002. Minimal and best linear combination of oxidative stress and antioxidant biomarkers to discriminate cardiovascular disease. Nutr Metab Cardiovasc.Dis. 12, 259-266.

Shekelle, P.G., Morton, S.C., Jungvig, L.K., Udani, J., Spar, M., Tu, W., Suttorp, J., Coulter, I., Newberry, S.J., and Hardy, M., 2004. Effect of supplemental vitamin E for the prevention and treatment of cardiovascular disease. J.Gen.Intern.Med. 19, 380-389.

Singh, R.B., Ghosh, S., Niaz, M.A., Singh, R., Beegum, R., Chibo, H., Shoumin, Z., and Postiglione, A., 1995. Dietary Intake, Plasma Levels of Antioxidant Vitamins, and Oxidative Stress in Relation to Coronary Artery Disease in Elderly Subjects. The American Journal of Cardiology 76, 1233-1238. 
Smith, G.D., Egger, M., and Phillips, A.N., 1997. Meta-analysis: Beyond the grand mean? BMJ 315, 1610.

Song, F., Sheldon, T.A., Sutton, A.J., Abrams, K.R., and Jones, D.R., 2001. Methods for Exploring Heterogeneity in Meta-Analysis. Eval Health Prof 24, 126-151.

Spoor, P., Airey, M., Bennett, C., Greensill, J., and Williams, R., 1996. Use of the capturerecapture technique to evaluate the completeness of systematic literature searches. BMJ 313, 342-343.

Steinbrecher, U.P., Parthasarathy, S., Leake, D.S., Witztum, J.L., and Steinberg, D., 1984. Modification of Low-Density Lipoprotein by Endothelial-Cells Involves LipidPeroxidation and Degradation of Low-Density Lipoprotein Phospholipids. Proceedings of the National Academy of Sciences of the United States of AmericaBiological Sciences 81, 3883-3887.

Sterne, J.A.C., Egger, M., and Smith, G.D., 2001. Systematic reviews in health care: Investigating and dealing with publication and other biases in meta-analysis. BMJ 323, 101-105.

Strobel, N.A., Fassett R.G., Marsh S.A., Coombes J.S., 2011. Oxidative stress biomarkers as predictors of cardiovascular disease International Journal of Cardiology 147, 191201.

Stroup, D.F., Berlin, J.A., Morton, S.C., Olkin, I., Williamson, G.D., Rennie, D., Moher, D., Becker, B.J., Sipe, T.A., Thacker, S.B., and for the Meta-analysis Of Observational Studies in Epidemiology Group, 2000. Meta-analysis of Observational Studies in Epidemiology: A Proposal for Reporting. JAMA 283, 2008-2012.

Sutton, A.J., Duval, S.J., Tweedie, R.L., Abrams, K.R., and Jones, D.R., 2000. Empirical assessment of effect of publication bias on meta-analyses. BMJ 320, 1574-1577.

Tamer, L., Sucu, N., Polat, G., Ercan, B., Aytacoglu, B., Yucebilgic, G., Unlu, A., Dikmengil, M., and Atik, U., 2002. Decreased serum total antioxidant status and erythrocytereduced glutathione levels are associated with increased serum malondialdehyde in atherosclerotic patients. Arch.Med.Res. 33, 257-260.

Thornton, A. and Lee, P., 2000. Publication bias in meta-analysis: its causes and consequences. Journal of Clinical Epidemiology 53, 207-216.

Turgan, N., Boydak, B., Habif, S., Gulter, C., Senol, B., Mutaf, I., Ozmen, D., and Bayindir, O., 1999. Urinary hypoxanthine and xanthine levels in acute coronary syndromes. Int.J Clin Lab Res. 29, 162-165.

Vivekananthan, D.P., Penn, M.S., Sapp, S.K., Hsu, A., and Topol, E.J., 2003. Use of antioxidant vitamins for the prevention of cardiovascular disease: meta-analysis of randomised trials. Lancet 361, 2017-2023.

Weinbrenner, T., Cladellas, M., Isabel Covas, M., Fito, M., Tomas, M., Senti, M., Bruguera, J., and Marrugat, J., 2003. High oxidative stress in patients with stable coronary heart disease. Atherosclerosis 168, 99-106.

Williams KJ, Fisher EA, 2005. Oxidation, lipoproteins, and atherosclerosis: which is wrong, the antioxidants or the theory? Curr Opin Clin Nutr Metab Care 8, 139 -146.

Witztum, J.L., 1994. The oxidation hypothesis of atherosclerosis. The Lancet 344, 793-795.

Witztum, J.L., 1998. To E or Not To E-How Do We Tell? Circulation 98, 2785.

Witztum, J.L. and Steinberg, D., 2001. The Oxidative Modification Hypothesis of Atherosclerosis; Does It Hold for Humans? Trends in Cardiovascular Medicine 11, 93-102. 


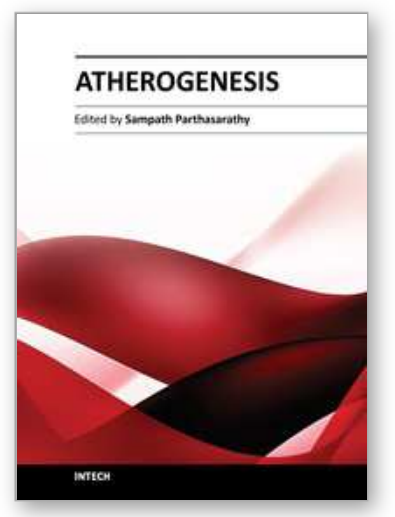

\author{
Atherogenesis \\ Edited by Prof. Sampath Parthasarathy
}

ISBN 978-953-307-992-9

Hard cover, 570 pages

Publisher InTech

Published online 11, January, 2012

Published in print edition January, 2012

This monograph will bring out the state-of-the-art advances in the dynamics of cholesterol transport and will address several important issues that pertain to oxidative stress and inflammation. The book is divided into three major sections. The book will offer insights into the roles of specific cytokines, inflammation, and oxidative stress in atherosclerosis and is intended for new researchers who are curious about atherosclerosis as well as for established senior researchers and clinicians who would be interested in novel findings that may link various aspects of the disease.

\title{
How to reference
}

In order to correctly reference this scholarly work, feel free to copy and paste the following:

Yedidya Dotan, Dov Lichtenberg and Ilya Pinchuk (2012). Are CVD Patients Under Oxidative Stress?, Atherogenesis, Prof. Sampath Parthasarathy (Ed.), ISBN: 978-953-307-992-9, InTech, Available from: http://www.intechopen.com/books/atherogenesis/are-cvd-patients-under-oxidative-stress-

\section{INTECH}

open science | open minds

\section{InTech Europe}

University Campus STeP Ri

Slavka Krautzeka 83/A

51000 Rijeka, Croatia

Phone: +385 (51) 770447

Fax: +385 (51) 686166

www.intechopen.com

\section{InTech China}

Unit 405, Office Block, Hotel Equatorial Shanghai

No.65, Yan An Road (West), Shanghai, 200040, China 中国上海市延安西路65号上海国际贵都大饭店办公楼405单元

Phone: +86-21-62489820

Fax: $+86-21-62489821$ 
(C) 2012 The Author(s). Licensee IntechOpen. This is an open access article distributed under the terms of the Creative Commons Attribution 3.0 License, which permits unrestricted use, distribution, and reproduction in any medium, provided the original work is properly cited. 